\title{
Special issue on activity-travel decision processes
}

\author{
Sean Doherty $\cdot$ Dick Ettema
}

(C) Springer Science+Business Media B.V. 2006

Before you lies the 2006 TRB (Transportation Research Board) Special Issue of Transportation comprising selected papers presented at the 2006 Annual Meeting of the TRB in sessions sponsored by the Travel Behavior and Values committee on activity-travel decision processes. We are pleased to serve as editors of this Special Issue. The Special Issue is the outcome of a partnership between Transportation and the TRB, a division of the National Research Council, which serves the National Academy of Sciences and the National Academy of Engineering in the United States. This partnership builds on TRB's commitment to build bridges that help disseminate cutting edge research and state-of-the-art solutions in transportation to a worldwide audience. It involves two standing committees of TRB's Division A (Technical Activities): the Committee on Traveler Behavior and Values ABD10) and the Committee on Travel Demand Forecasting (ADB40).

Over the past decades, we have witnessed a gradual development of travel behaviour theory, involving the consecutive introduction of various paradigms. In the early days, traffic flows, resulting from individuals' travel decisions, were characterised as analogies to physical processes such as gravity theory (for modelling trip generation and distribution) or queuing theory (for modelling traffic flows in networks). This practice was typically based on a conceptualisation of traffic as an aggregate phenomenon, thought of in terms of amounts and flows.

The introduction of discrete choice models in the seventies focused attention on individuals as decision-making units underlying aggregate traffic flows. Although later extensions of discrete choice models show a trend towards integration, they typically describe how individuals make decisions regarding single (or a few) facets of their travel behaviour, such as the mode or destination of a particular trip. These

\footnotetext{
S. Doherty $(\bowtie)$

Department of Geography and Environmental Studies, Wilfrid Laurier University, Waterloo, Ontario, Canada N2L 3C5

e-mail: sdoherty@wlu.ca

D. Ettema

Faculty of Geosciences, Utrecht University, 3508 TC Utrecht, The Netherlands e-mail: d.ettema@geo.uu.nl
} 
decisions are assumed to be rational in the sense that individuals choose the alternative that maximises their expected utility.

The next paradigm shift occurred with the gradual introduction of the activitybased approach to travel decision-making. Activity based theory focuses on aspects of travel decision making that are less well addressed in discrete choice theory. In particular, the relationship between various activities throughout the day is emphasized. Much research has also been carried out into the activity scheduling decision process that gives rise to observed activity-travel patterns. This especially concerns how individuals select, plan, execute and adapt their activities over time, space and across individuals. An important motivation for doing so has been the idea that activity participation is the main trigger of travel, and that by understanding decisions about activity participation and planning, a better insight is gained in travel decision-making.

Since the introduction of the activity-based approach in the early eighties, it has resulted in a host of travel models and new insight into travel behaviour. Current research on travel behaviour aims both at refining the existing concepts and models and at charting new theoretical concepts. This Special Issue aims at presenting work in both streams. The papers reflect in particular the continuing efforts of TRB committees to encourage travel behaviour researchers to adopt insights from other fields in our efforts to better understand the unique nature of activities and the decision processes that give rise to them.

A paper in the first stream is the one by Ruiz and Timmermans. They observe that, although activity-based models have been well established over the past decade, models of activity rescheduling are still scarce. Their paper contributes to filling this gap by focusing on a particular instance of rescheduling: the case where a single new activity episode is inserted between two existing consecutive pre-planned activities. Their analysis is based on an Internet survey held in Valencia, Spain in 2003, in which respondents reported how pre-planned scheduled were modified during execution. Hazard models were estimated in which the change in the duration of the anterior and posterior activity were the dependent variables. Independent variables were socio-demographics and characteristics of the pre-planned schedule. The only socio-demographic that appeared to influence the rescheduling was gender, with males changing the timing of the posterior activity more than females. It was further found that activities scheduled at a later time of the day are less subject to retiming, and that leisure and social activities are modified less than for instance attending or giving a lecture. Also the duration of both the existing and the new activities impact on the amount of retiming. Finally, posterior out-of-home activities undergo smaller changes in duration than in-home activities, and flexible posterior activities undergo larger changes in duration. Based on these findings, the authors conclude that there exists a need for the simultaneous modelling of the duration of anterior and posterior activity durations in the context of the aforementioned insertions in rescheduling processes.

The other four papers in this issue address the upcoming topic of the role of social networks and social interaction in the travel decision-making process. In this respect they aim at a fundamental extension of travel theory: whereas the activity-based approach assumes that activities are the drivers of travel, these papers try to identify what inherently drives participation in certain activities. This seems to be especially important in the context of social activities, which transportation researchers continue to find difficult to understand and predict. A common assumption underlying 
these papers is that participation in activities is driven by roles that we fulfil in networks of family, friends, colleagues or otherwise. The motivation to engage in certain activities may spawn from our role in various social networks and from the composition of these networks. Also, when implementing activity participation decisions, the social network may play an important role through the location of other members of the social network with whom we want to engage in activities or because we need to negotiate with others over limited resources. Finally, the social network may serve as a source of information about activities, locations and circumstances. Thus, by being active in our social network we will learn about opportunities and limitations offered by the environment, which may further trigger activities and trips.

It is noted that research on the various relationships between travel, activities and social networks has just started to develop. The papers included in this Special Issue are examples of such research and explore various aspects of the relationship between social interaction and activity/travel behaviour. A fundamental approach to this topic is taken by Goulias and Henson, who investigate underlying motivations for activity participation. Their paper builds on recent research into human motivations for action that emphasizes that both ego and altruism are important motivations for engaging in activities. They further investigate the effect of these motivations based on an activity diary of 1471 persons in Pennsylvania, in which respondents reported not only the activities they engaged in but also 'with whom' and 'for whom' they conducted the activity. Using latent class cluster analysis, individuals are classified into classes that spend more or less time on altruistic or self-serving behaviour, and these classes appear to coincide well with social roles. For instance, it is found that children often appear in clusters with a high degree of egoistic behaviour, whereas household heads also appear in cluster with more altruistic behaviour. On the household level it is found that spouses may belong to different egoistic or altruistic clusters, but the most frequent $(53 \%)$ combination is that spouses both fall into one of the altruistic clusters, suggesting balanced reciprocity within the household. Another striking observation is that out-of-home activities are more often conducted for altruistic reason than in-home activities. Over all, Goulias and Henson's work suggests that role specific motivations may play an important role in decisions regarding activity participation and travel.

Carasco and Miller's paper focuses on the decision to perform a social activities, as characterized by the individual's "propensity" to engage in a social projects which depends upon: personal attributes, their social network composition (how many, their tie strength, and frequency/media of interaction), and the on-going history of interactions and opportunities to engage in a social episodes. They further suggest that this propensity is a "latent" attribute, not directly observable from individuals' activity patterns (and hence from traditional diary data). Given this, they present a new type of data collection instrument designed to explore the various aspects of social network, and use the data to calibrate a model of the propensity to perform social activities by each type and strength of tie. Although they see this as the start of an innovative new approach, they were able to discover interesting new effects related to communication and information technology use, social activity types, and the role of physical space. Their work suggests that composition of the social network may contribute especially to understanding and predicting individuals' engagement in social activities and the associated travel. 
A more specific view on social interaction is taken by Hollander and Prashker, who focuses on interaction and negotiation processes between multiple actors, such as described in game theory. The central contention of this paper is that travel decisions are not made by individuals in isolation, but keep in mind the potential outcome of other individuals' or authorities' decisions, on which one can anticipate. The paper reviews various studies of games between travellers. In many cases, these games involve competition between travellers for limited resources, in which travellers may follow different strategies (e.g. hawks against doves). An important conclusion is that most games involving multiple travellers lead to suboptimal outcomes due to the so called prisoners dilemma: all players would be better off if they did not act selfishly, but individual travellers would then be able to improve their situation by acting selfishly. With respect to games between travellers and authorities, the paper discusses examples where road authorities manipulate signalling regimes or toll regimes such as to maximise revenues or minimise congestion, whereas individual travellers aim at minimising their generalised travel costs. In most cases, the authority is regarded as the leading player, and the travellers as followers. This leads to bi-level Stackelberg games, in which the authority solves an optimisation problem with equilibrium constraints. Overall, Hollander and Prashker's paper makes clear that interaction between various decision makers is a relevant topic, especially in cases where they compete for scarce commodities such as time, road capacity or other resources.

Srinivasan and Bhat, finally, focus their research on interactions on the household level. Building on earlier work in the area of household decision-making (see the 2005 TRB Special Issue of Transportation, Volume 32, No 5) they present a comprehensive and econometrically advanced model of the participation and duration in discretionary in-home and out-of-home activities, and on the decision to pursue such activities individually or jointly. Both the participation and duration can principally be affected by socio-demographics, weekly and seasonal variations and the need to engage in mandatory and maintenance activities. The model was calibrated using the 2000 San Francisco Bay Area Travel Survey. With respect to participation, the model suggests that apart from socio-demographics and seasonal effects, the composition of the activity pattern plays an important role. For instance, if more time is spent on in-home work, individuals are less likely to undertake in-home and outof-home discretionary activities, while those working out-of-home are less likely to undertake joint discretionary activities. With respect to activity durations, trade-offs are found between mandatory activity time (in-home and out-of-home) and discretionary activities, and between maintenance time (in-home and out-of-home) and discretionary activities. Various error terms included in the model indicate positive correlations between male and female in-home and out-of-home participation in discretionary activities. Another finding in this respect is that with increasing participation in in-home discretionary activities, the participation in out-of-home joint activities decreases, suggesting a trade-off between these categories. The paper includes an application of the model to evaluate TDM (Travel Demand Management) strategies, which makes clear that the model can predict a richer gamma of responses than more conventional travel demand models, in particular with respect to time-use and household interactions.

The papers included in this Special Issue make a strong contribution to a better understanding of activity-travel decision processes, especially in the context of activity rescheduling and social interaction. Nevertheless, many issues still remain to 
be explored in these areas of research. With respect to activity rescheduling, more comprehensive models need to be developed that cover cases of activity rescheduling, such as deleting, replacing and modifying activities. Calibrating such models is likely to create a variety of challenges in terms of data collection and statistical techniques. In particular, new types of activity attributes that capture the spatial, temporal and interpersonal flexibility/fixity of activities are likely needed to predict what activities are chosen for rescheduling and how so. Explicitly measuring/ modelling these would avoid the often unrealistic assumptions adopted in most applied models that certain activity types (e.g. work, shopping, joint activities) share the same level flexibility for all persons in all situations. How rescheduling fits into the larger hierarchy of scheduling decisions is also a key future concern. With respect to social interaction and social networks, we are witnessing the initial stage of a research area, involving many coevolving paradigms and methodologies. Serious effort will need to be given to define more comprehensive approaches, particularly focussing on the overlapping parts of the existing studies. For example, the relationship between the household and the wider social network, the various functions of such networks (information dissemination, negotiation, activity initiation), and their mutual coherence. This will likely be an extremely challenging area for innovative studies in the years to come.

To, conclude we wish to acknowledge the efforts of Mark Norman and Kimberly Fisher of the TRB, TRB Division A standing committee chairs Ram Pendyala (ADB10) and Chandra Bhat (ADB40), and Editor-in-Chief of Transportation Martin Richards, in making this Special Issue possible. In addition to the papers published in this Special Issue, numerous papers submitted to the TRB Committees for publication consideration appear annually in the Transportation Research Record: Journal of the Transportation Research Board series. These papers collectively represent major contributions that advance the state-of-the-art in activity-travel behaviour modeling and demand forecasting.

\section{About the authors}

Sean Doherty is an Associate Professor of Geography and Environmental Studies and holds a PhD in Civil Engineering. He is chair of the TRB subcommittee on Behavioral Processes, sits on the board of the International Association of Travel Behaviour Research, and recently received the 2006 Fred Burggraf Award from TRB recognizing excellence in transportation research.

Dick Ettema is an Assistant Professor in the Faculty of Geosciences at Utrecht University in The Netherlands. His research interests center around the analysis and modelling of activity patterns, including the impact of land use on activity scheduling, household interactions and longer term adaptation processes. He is member of the TRB Committee on Traveler Behavior and Values and co-chair of the Subcommittee of Time Use and Activity Patterns. He is Director of Education of the Netherlands Graduate School of Urban and Regional Research (NETHUR). 